\title{
Construction of Control Charts for Monitoring Various Parameters Related to the Management of the COVID-19 Pandemic
}

\author{
Mame Faty Mbaye, Ngor Sarr, Baba Ngom* \\ Laboratoire Sciences et Techniques de l'Eau et de l'Environnement (LASTEE), Ecole Polytechnique de Thiès, Thiès, Sénégal \\ Email: *bngom@ept.sn
}

How to cite this paper: Mbaye, M.F., Sarr, N. and Ngom, B. (2021) Construction of Control Charts for Monitoring Various Parameters Related to the Management of the COVID-19 Pandemic. Journal of Biosciences and Medicines, 9, 9-19.

https://doi.org/10.4236/jbm.2021.93002

Received: January 27, 2021

Accepted: March 13, 2021

Published: March 16, 2021

Copyright (c) 2021 by author(s) and Scientific Research Publishing Inc. This work is licensed under the Creative Commons Attribution International License (CC BY 4.0).

http://creativecommons.org/licenses/by/4.0/

\begin{abstract}
To monitor the quality characteristics of a process, appropriate graphical and statistical tools must be used. These tools are capable of showing the evolution over time of the behavior of the quality characteristics (measurable or countable) and detecting situations that seem to present certain anomalies. The control chart is one of these tools widely used in quality management. In the process of managing the COVID-19 pandemic, this tool will make it possible to know at all times whether the parameters monitored such as the positivity rate, the recovery rate, and the mortality rate, are under control and to act accordingly. Monitoring cure and mortality rates will also show us the effectiveness of the treatments used.
\end{abstract}

\section{Keywords}

COVID-19, Statistical Process Control, Control Charts, Healthcare

\section{Introduction}

The health crisis linked to the COVID-19 pandemic which outbroke in December 2019 in China has raised political, economic, and above all medical challenges in Senegal and around the world [1] [2] [3]. Most countries have taken bold quarantine and unprecedented lockdown measures to control the spread of COVID-19. Despite those rigorous global containment and quarantine efforts, the incidence of COVID-19 continues to rise, with over 95,000,000 laboratory-confirmed cases and over 2,100,000 deaths worldwide, as of January 25, 2021. How individual countries politics and health systems respond to the ongoing pandemic will be critical in influencing the trajectory of national epidemics. About the responses against the novel coronavirus disease, we mainly focus on 
financial aspect. Of course, investments are necessary to build strong health care systems, and to face public health challenges like that linked with COVID-19, however with little interest toward quality, all efforts may be ineffective.

The management of the health system, the care of patients by hospitals, and the evaluation of the strategies implemented have now become central and require methodical management.

Quality tools such as Statistical Process Control (SPC) techniques have played an efficacious role in monitoring hospital performance such as mortality rate, pre and post-operative complications, number of infections in hospital etc. [4] [5]. SPC and its primary tool-the control chart-provide researchers and practitioners with a method of better understanding and communicating data from healthcare improvement efforts [6]. Thus, the use of statistical process control (SPC) seems well suited to the management of the pandemic by continuous monitoring of indicators for a dynamic improvement of the control strategies implemented by the political authorities, and healthcare practitioners.

The process approach must be favored in health whenever we wish to improve the quality of patient care and keeping the situation under control. The precise analysis of the studied process is also essential for the definition of relevant indicators.

The SPC method, an advanced self-checking technique, is based on three fundamental principles [7] [8] [9]:

- The priority is given to prevention (intervention before a loss of control of the situation).

- The reference to the process as it works (qualification of the health system).

- The empowerment and active participation of stakeholders.

Our objective through this article is to provide solutions to the problem of managing the pandemic, particularly in Senegal, through the piloting maps of the SPC.

The SPC allows the identification of the type of behavior of an indicator: predictable (common causes) or unpredictable (special causes).

The common causes are the many sources of variations attributable to chance which are always present to varying degrees in different processes. These causes are always present and more and more will require "living with". All of these common causes form the intrinsic variability of the process.

Special causes are identifiable causes of dispersion, often irregular and unstable, and therefore difficult to predict. The appearance of a special cause requires intervention in the process. Unlike common causes, special causes are generally few. In the context of pandemic management, a special cause such as an abrupt increase in the number of positive cases or the number of cured patients must question health personnel about the potential causes (unreliability of tests, non-compliance with preventive measures, treatment effectiveness).

This work gave insight into the use of statistical control process in managing pandemic outbreak. It provided also a solid basis for future works aimed to in- 
vestigate the implementation of quality tools to improve healthcare management efficiency in developing countries.

\section{Materials and Methods}

\subsection{Types of Control Charts}

\section{We have two types of control charts:}

> Measurement control charts "X-E/R".

$>$ The control charts by attribute "p" et "c".

The "Average (X)-Range (E/R)" chart is made up of two charts: one for the average and the other for the range. The reason for using these two variables is that using just average can sometimes be insufficient to explain a deviation from a process.

The two parameters to monitor in an X-E/R map are position (X) and dispersion $(\mathrm{E} / \mathrm{R})$. These two control charts must therefore be designed.

These control charts apply only to the control of variables by measures [10].

The method of quality control by attributes essentially consists of noting the presence or absence of any qualitative criterion (attribute) on each controlled unit and compiling the number of units having or not having this criterion on all of the checked units. Thus, in the case of pandemic management, instead of counting the number of infected, we will only have to calculate the positivity rate compared to the number of people tested, and check if this rate is inside control and/or monitoring limits.

The main charts for control by attributes are:

- p chart: which checks the proportion of non-compliant (positive cases, deaths);

- $n p$ chart: which controls the number of non-compliant units;

- c chart: which checks the number of non-conformities (comorbidities from which a patient suffers);

- $\mathrm{u}$ chart: which checks the average number of non-conformities by sub-group (children, young people, and the elders).

Figure 1 summarizes these different types of control charts.

\subsection{Control Chart Choice}

For this present study, the " $\mathrm{p}$ " chart seems the most suitable for monitoring the positivity rate which is an indicator of control of the situation, the recovery rate, and the mortality rate which are indicators of the effectiveness of the treatments. However, chart $\mathrm{c}$ would be indicated for a more detailed analysis of mortality, but this requires the availability of data on the various pathologies of deceased patients. The $\mathrm{u}$ chart could also be useful for monitoring different groups (ages, sexes).

A chart $\mathrm{p}$ (or chart for the proportion of non-compliant or defective) is a graph showing the proportion of non-compliant observed in each sample according to the sampling times (time). The average proportion of non-conformers as 


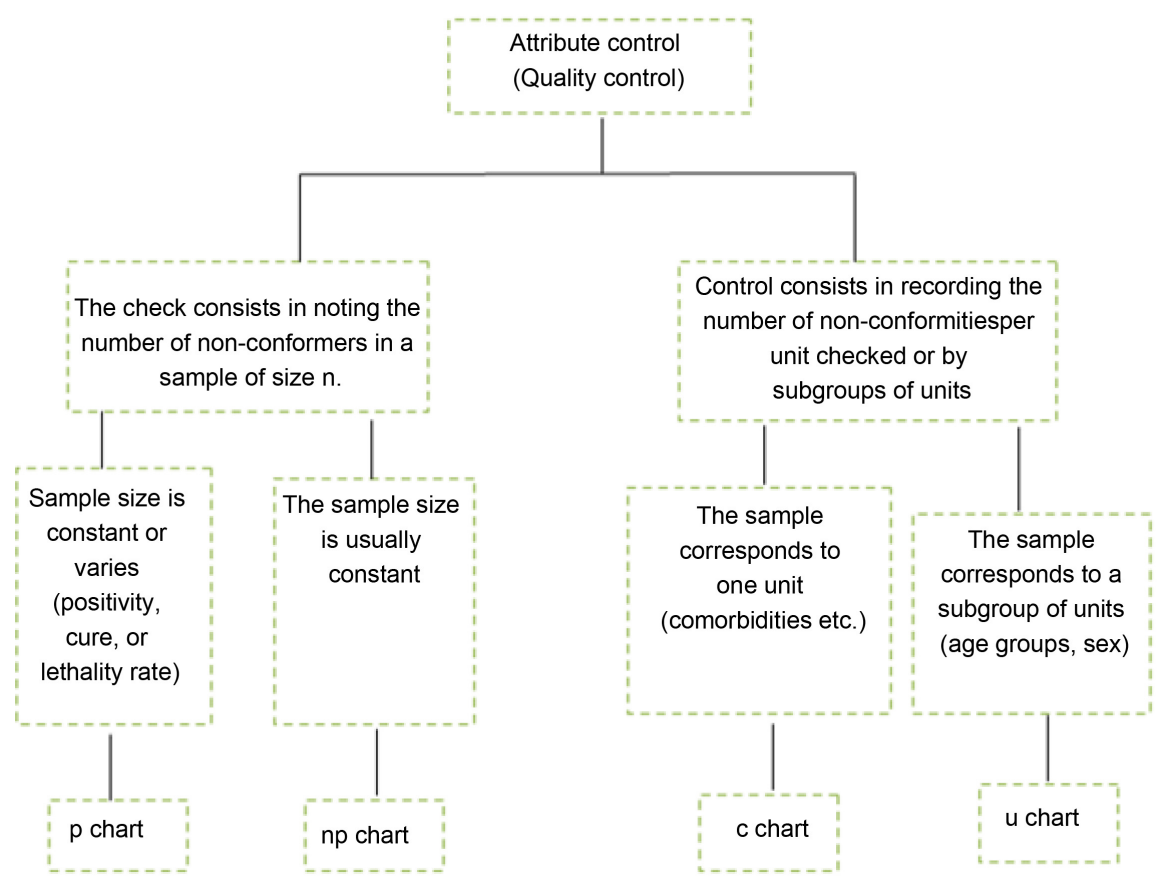

Figure 1. Different types of control charts by attributes [11].

well as the limits of upper and lower control. Figure 2 shows the shape of most $\mathrm{p}$ charts.

$$
\bar{p}-3 \sqrt{\frac{\bar{p}(1-\bar{p})}{\sqrt{n i}}} \text { and } \bar{p}+3 \sqrt{\frac{\bar{p}(1-\bar{p})}{\sqrt{n i}}} \text { are called respectively lower control }
$$

limit (LCLp) and upper control limit (UCLp) of the chart.

$$
\bar{p}-2 \sqrt{\frac{\bar{p}(1-\bar{p})}{\sqrt{n i}}} \text { and } \bar{p}+2 \sqrt{\frac{\bar{p}(1-\bar{p})}{\sqrt{n i}}} \text { are named respectively lower sur- }
$$

veillance limit (LSLp) and upper surveillance limit (USLp) of the chart.

\subsection{Control Chart Implementation}

The implementation of a control chart for the proportion of non-conformities is relatively simple and the different steps of its construction are given in Figure 3.

We will add a solid green line as the objective line corresponding to $100 \%$ for the cure rate and $0 \%$ for positivity and mortality. The LSCs can be represented by solid thin lines, which may facilitate the exploitation of the chart.

\subsubsection{Sample Size}

For this type of control to be effective, it is necessary that the workforce is large enough. Usually, a sample size (subgroup) between 50 and 200 units (or more) is required. Whenever possible, the sample size is kept constant for all samples.

\subsubsection{Control Frequency}

Sampling frequency should allow monitoring realistic production periods so that the analysis of the map leads to an appropriate diagnosis of quality problems and that corrective measures are applied as quickly as possible. 


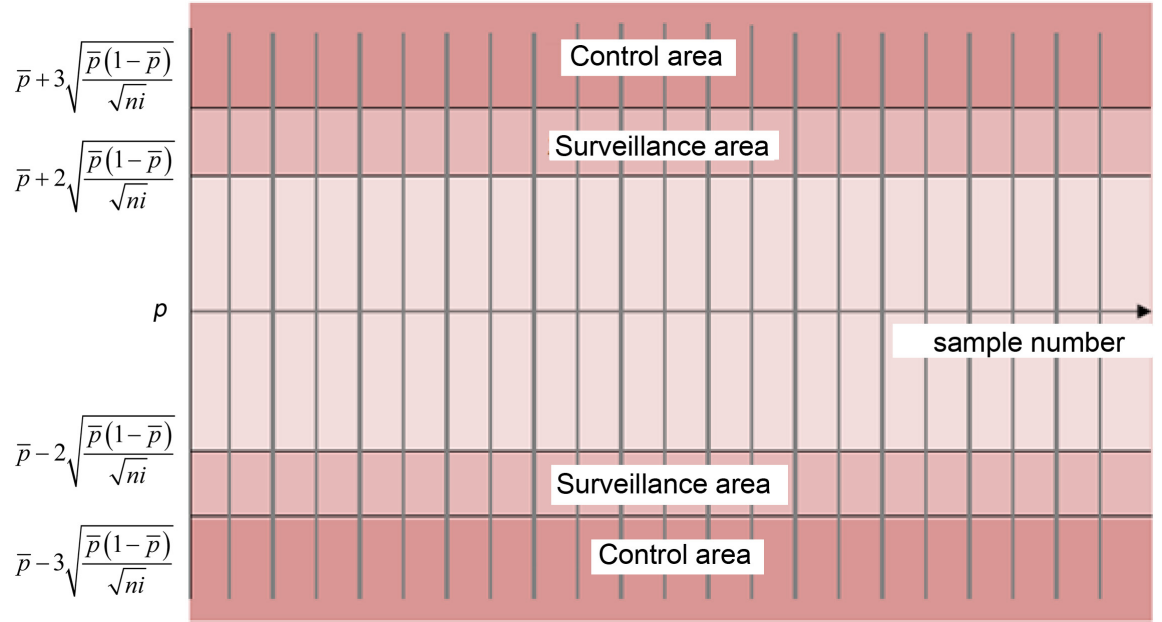

Figure 2. Shape of most $p$ charts.

\section{Sampling procedures}
a) Sample size
b) Frequency control
c) Number of samples to be taken

Calculation of proportions $p$

Calculation of the proportion of non-compliant (or defective) for each sample

\section{Graphical representation of proportions}

Transfer the values obtained in 2 to the control card, indicating on the ordinate (vertical axis) the proportion (or \%) of non-conformities and on the abscissa (horizontal axis) the identification of the sample (hours, days, etc.). Connect the dots together

3

4

\section{Calculation of control limits}

a) Calculation of the average proportion of non-conformers

b) Calculation of upper and lower limits of control

5

b) Horizontal dotted lines for upper and lower control limits

c) The values of $p$, UCLp and LCLp are usually indicated on the map $p$

Identification of out of control points and other specific behaviors

Figure 3. Different steps of developing a control chart. 


\subsubsection{Calculation of Proportions $p$}

The number of non-conforming units is recorded for each sample of size $n$. The proportion $p$ of non-conforming is obtained from:

$$
p=\frac{\text { Number of nonconformities in the sample }}{\text { Sample size }}=\frac{d}{n}
$$

\subsubsection{Graphic Representation of Proportions}

It is a question of plotting on the control chart the values obtained for the proportions of nonconforming, these values being associated with the vertical axis on the identification of the subgroup (sample) corresponding to each $p$-value being plotted on the horizontal axis. The graduation of the vertical axis is between $0 \%$ and $100 \%$.

It is also common practice to connect the points on the map together to facilitate the analysis of the behavior of the proportion of non-conformers.

\subsubsection{Calculation of Control Limits}

- Upper control limit

$$
\mathrm{UCL}=\bar{p}+3 \sqrt{\frac{\bar{p}(1-\bar{p})}{\sqrt{n}}}
$$

- Lower control limit

$$
\mathrm{LCL}=\bar{p}-3 \sqrt{\frac{\bar{p}(1-\bar{p})}{\sqrt{n}}}
$$

The monitoring limits are two standard deviations on each side of the average proportion:

- Upper surveillance (monitoring) limit

$$
\mathrm{USL}=\bar{p}+2 \sqrt{\frac{\bar{p}(1-\bar{p})}{\sqrt{n}}}
$$

- Lower surveillance(monitoring) limit

$$
\mathrm{LSL}=\bar{p}-2 \sqrt{\frac{\bar{p}(1-\bar{p})}{\sqrt{n}}}
$$

\subsubsection{Diagnostics}

The objective of the control chart is to monitor the behavior of a statistic variable (here, a proportion) over time and to be able to identify the abnormal fluctuations. We will admit that the process is mastered if no point is outside the control limits or if the arrangement of the points does not reveal a trend or a series of points which would translate into a gradual drift and if nothing is done, the characteristic will go out of control limits. Surveillance limits, on the other hand, are warning signs: anything that comes out of it must call for vigilance [12].

\subsubsection{Chart p When the Sample Size Varies}

If the sample size (or subgroup size) varies significantly from one sample to another, then the control limits must be calculated for each sample (theoretical- 
ly, the control limits should be calculated for each size when this is not constant; however, this leads to a control chart with a visual aspect that is difficult to interpret).

The average proportion is always calculated in the same way, that is:

$$
\bar{p}=\frac{\text { total number of nonconformities }}{\text { total number of inspected units }}=\frac{\sum d_{i}}{\sum n_{i}}
$$

The control limits for each sample are then:

- Lower control limit (LCLp)

$$
\operatorname{LCLp}=\bar{p}-3 \sqrt{\frac{\bar{p}(1-\bar{p})}{\sqrt{n i}}}
$$

- Upper control limit (UCLp)

$$
\mathrm{UCLp}=\bar{p}+3 \sqrt{\frac{\bar{p}(1-\bar{p})}{\sqrt{n i}}}
$$

And the surveillance limits are as follows:

- Lower surveillance limit (LSLp)

$$
\operatorname{LSLp}=\bar{p}-2 \sqrt{\frac{\bar{p}(1-\bar{p})}{\sqrt{n i}}}
$$

- Upper surbeillance limit (USLp)

$$
\mathrm{USLp}=\bar{p}+2 \sqrt{\frac{\bar{p}(1-\bar{p})}{\sqrt{n i}}}
$$

where $\bar{p}$ is the average positivity rate and $n i$ is the sample size for the day, which means that the control limits are updated as the sample is variable.

\subsection{Process Behavior Analysis: Diagnostics}

The purpose of drawing a control chart, regardless of the character that is the subject of control, is to identify any behavior that could be identified as an uncontrolled situation and to take the necessary corrective measures. Uncontrolled situations are usually grouped into three categories [13]: 1) Points outside the control limits, 2) Continuation or trend (drifts) and 3) Other non-random behaviors.

- Points outside the control limits

Any point outside the control limits can be attributed to an abnormal situation, the cause of which must be found. We can use the $5 \mathrm{M}$ method in particular as diagnostic tools.

According to this method, the anomalies of a process come mainly from the 5 factors Material, Matter, Labor, Method, and Medium (5M).

- Point or trend sequences

An out of control situation can also resemble a series of points sensing a trend towards or out of the control limits.

A series of points above the average of the process is often attributable to con- 
ditions which have deteriorated or that the quality criteria have been changed; a series of points below the process average may be an indication that the conditions have changed for the better or that there has been a loosening in the control method or that there has been a change in the quality criteria. In this situation, the " $5 \mathrm{M}$ " analysis tool can be used to identify potential causes. A trend towards the objective line shows that the situation is going in the right direction [14] [15] [16].

\section{Results and Discussions}

\subsection{Political Management by the Positivity Rate}

The positivity rate is the number of positive tests out of the number of tests performed. We calculated the daily positivity rate over the period from March 22 to June 07,2020 , i.e. for a period of 78 days. Since the sample size (the number of tests) is variable, the most suitable chart is the $\mathrm{P}$ chart with a variable sample. The control limits will be: Equation (1) and Equation (2). Figure 4 shows the daily positivity rate.

The positivity rate fluctuates around the average. The limits of control being variable, we will make the interpretation with respect to the limit of the day, which will allow us to see if the situation is under control or not every day. Here we see that the situation today compared to this study is in SATU QUO: no way out of the crisis or worsening of the situation. The positivity rate is moving away from the upper control limit as of June 07, which shows that the situation is still under control.

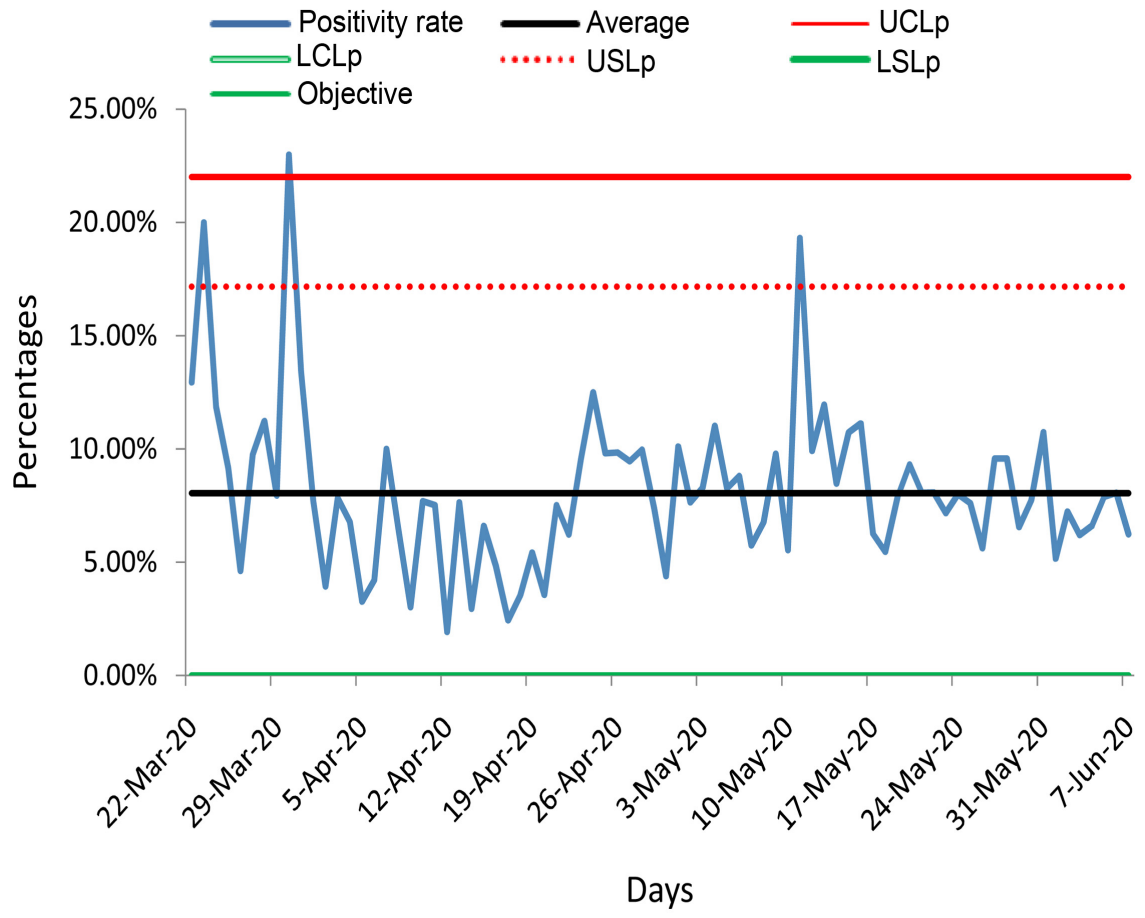

Figure 4. Evolution of the COVID-19 positivity rate over time. 


\subsection{Application to Cure Rate}

The cure rate is the number of patients declared cured out of the total number of infected. We calculated the daily cure rate over the period from March 21 to June 07,2020 . Since the sample size (the number of infected) is variable, the most suitable chart is the $\mathrm{P}$ chart with a variable sample. Figure 5 below shows changes in cure rate over time.

There is an irregular evolution of the cure rate and then an increase until reaching maximums between April 12 and 20. This period corresponds to the generalization of chloroquine for the treatment of COVID-19 for Senegal.

The cure rate then decreases and crosses the lower limit: this is explained by the fact that the number of tests carried out increased considerably from April 29,2020 . And at the beginning of May, there is a regular increase in the number of cured, which can be explained by the effectiveness of the treatments used.

It should be noted that in this context, what we are looking for is the fact that the characteristic studied (cure rate) exceeds the upper limit of control or approaches it. This means that the treatments are effective and this chart is an excellent monitoring tool for medical personnel.

\subsection{Application to the Case of Fatality Rate}

The fatality rate is the number of patients who died out of the total number of infected people. We have calculated the daily fatality rate over the period from March 21 to June 07, 2020. Since the sample size (the number of infected) is variable, the most suitable chart is the $\mathrm{p}$ chart with a variable sample. Figure 6 illustrates the evolution of the fatality rate.

The mortality rate is relatively low and is closer to the lower limit. However, a slight increase has been observed in the latter. This may be due to the increase in

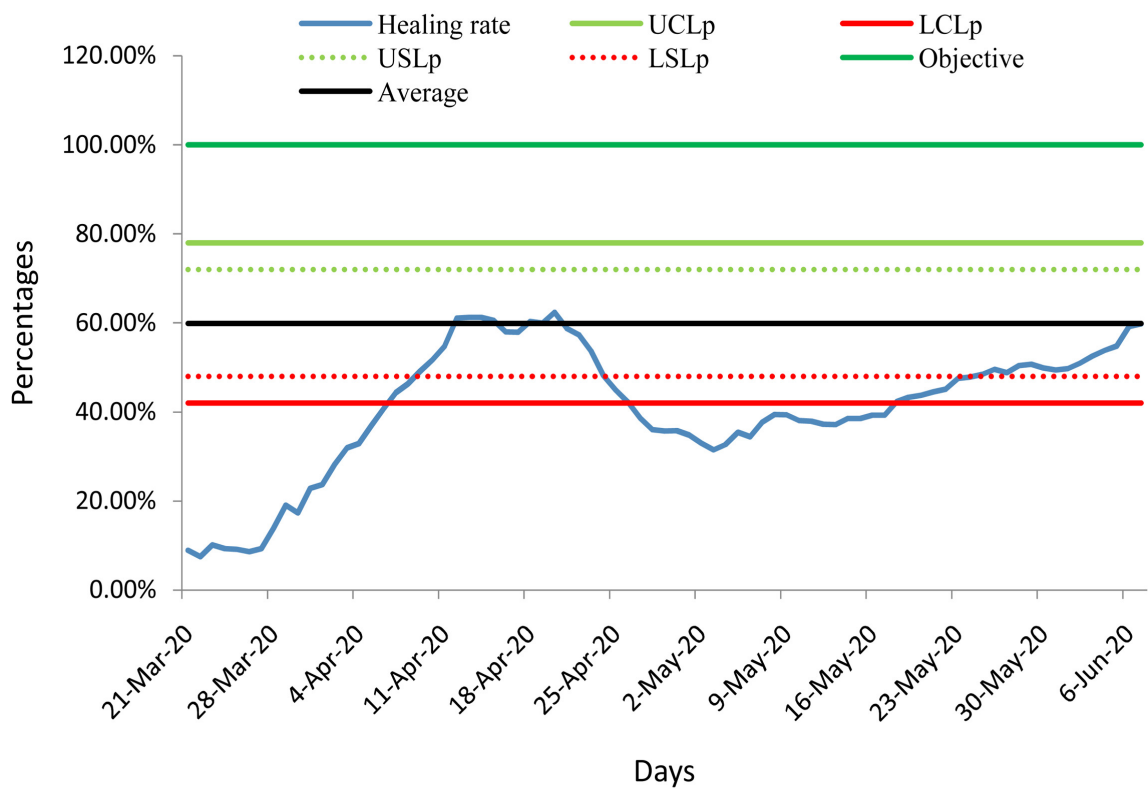

Figure 5. Healing rate evolution over time. 


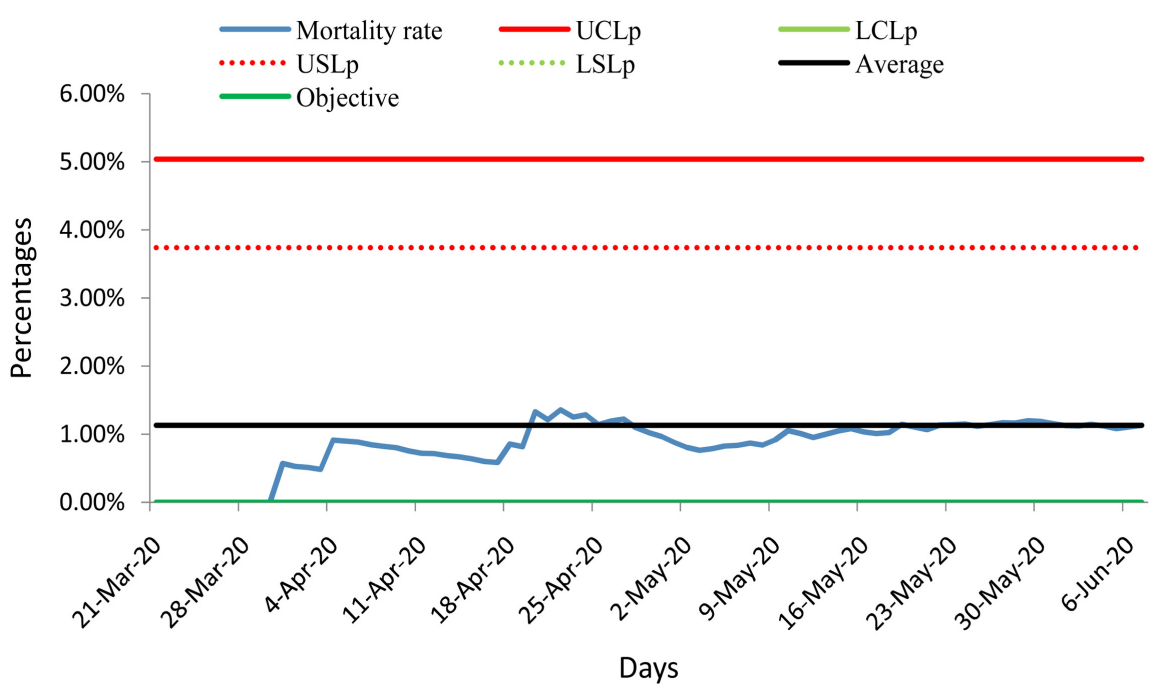

Figure 6. Mortality rate evolution over time.

the number of cases, which leads to an increase in the number of serious cases and therefore the number of deaths.

\section{Conclusion}

The objective of implementing the SPC method is to put a process under control. The first check to be carried out is to measure that the ability of the pandemic management process is good. If it is not the case, then it must be modified and improved. This step will significantly improve the dispersion of the process. It allows the manager to always look for new special causes, to remove them, and thus to improve the capability of the process. From improvement to improvement, the dispersion of the process will decrease and we can pretend to control the evolution of the pandemic. The primary objective of putting a process under control is therefore to make it predictable.

\section{Acknowledgements}

This work was supported by the Ministry of Higher Education, Research and Innovation of Senegal Republic through the COVID-19 fund.

\section{Conflicts of Interest}

The authors declare no conflicts of interest regarding the publication of this paper.

\section{References}

[1] Ozili, P.K. (2020) COVID-19 in Africa: Socioeconomic Impact, Policy Response and Opportunities. SSRN Electronic Journal. https://doi.org/10.2139/ssrn.3574767

[2] Walker, P.G., et al. (2020) The Global Impact of COVID-19 and Strategies for Mitigation and Suppression. Imp. Coll. COVID-19 Response Team.

[3] Cheval, S., Adamescu, C.M., Georgiadis, T., Herrnegger, M., Piticar, A. and Legates, 
D.R. (2020) Observed and Potential Impacts of the Covid-19 Pandemic on the Environment. International Journal of Environmental Research and Public Health, 17, 4140. https://doi.org/10.3390/ijerph17114140

[4] Benneyan, J.C. (1998) Statistical Quality Control Methods in Infection Control and Hospital Epidemiology, Part II: Chart Use, Statistical Properties, and Research Issues. Infection Control \& Hospital Epidemiology, 19, 265-283. https://doi.org/10.1086/647807

[5] Suman, G. and Prajapati, D.R. (2018) Control Chart Applications in Healthcare: A Literature Review. International Journal of Metrology and Quality Engineering, 9, 5. https://doi.org/10.1051/ijmqe/2018003

[6] Benneyan, J.C., Lloyd, R.C. and Plsek, P.E. (2003) Statistical Process Control as a Tool for Research and Healthcare Improvement. Quality \& Safety in Health Care, 12, 458-464. https://doi.org/10.1136/qhc.12.6.458

[7] Pillet, M. (2005) Appliquer la maîtrise statistique des procédés MSP/SPC.

[8] Renault, I. and Chéroute, G. (2006) Maîtrise statistique des processus Utilisation des cartes de contrôle. Tech. l'ingénieur.

[9] Jelali, M. (2013) Statistical Process Control. In: Jelali, M., Ed., Control Performance Management in Industrial Automation, Springer, Berlin, 209-217. https://doi.org/10.1007/978-1-4471-4546-2_8

[10] Thor, J., et al. (2007) Application of Statistical Process Control in Healthcare Improvement: Systematic Review. Quality and Safety in Health Care, 16, 387-399. https://doi.org/10.1136/qshc.2006.022194

[11] Baillargeon, G. (2017) Introduction aux méthodes statistiques en Application dans divers secteurs industriels. $4 \mathrm{e}$ ed.

[12] Maurice, M.M. (2013) L'amelioration de la qualite des soins de l'HMIMV de RABAT.

[13] El, M. and Falloul, M. (2015) Construction d'une carte de contrôle des procédés de production d'une société industrielle [Construction of a Control Chart of Production Processes of an Industrial Company]. International Journal of Innovation and Scientific Research, 13, 580-590.

[14] Tiplica, T. (2012) Des processus industriels multivaries. Spécialité: Sciences et Technologies Industrielles.

[15] Electric, W. (1965) Statistical Quality Control Handbook. Western Electric Co., Indianapolis.

[16] Alioua, A. (2012) La Revue Annuelle Qualite du Produit: Une démarche managériale vers une extension normative ISO 9001. 